\title{
Use of an intense microwave laser to dissociate a diatomic molecule: Theoretical prediction of dissociation dynamics ${ }^{\#}$
}

\author{
AMITA WADEHRA $^{\mathrm{a}, \S}$ and B M DEB ${ }^{*, \mathrm{~b}}$ \\ ${ }^{a}$ Department of Chemistry, Panjab University, Chandigarh 160 014, India \\ $\S$ Present address: Department of Physics, Ohio State University, Columbus, OH 43210, USA \\ ${ }^{b}$ Department of Chemical Sciences, Indian Institute of Science Education and Research-Kolkata, \\ Mohanpur Campus, P.O. BCKV Main Campus, Mohanpur 741 252, India \\ e-mail: bmdeb@yahoo.co.in
}

\begin{abstract}
By employing an intense microwave laser of wavelength $116.65 \mu \mathrm{m}$ with intensities $1 \times 10^{13}$ and $5 \times 10^{18} \mathrm{~W} \mathrm{~cm}^{-2}$, respectively, the conclusion is reached theoretically and computationally that it is possible to dissociate the $\mathrm{CO}$ molecule, modelled as a Morse oscillator. It is predicted that for above-threshold dissociation (ATD), the molecule should absorb 1044 photons of the given wavelength in order to reach the lowest edge of the vibrational continuum. A consistent analysis of the predicted dissociation process is provided though the time-dependent probability density, dissociation probability, norm, potential function, HHG and ATD spectra, obtained by numerically solving the time-dependent vibrational Schödinger equation.
\end{abstract}

Keywords. Above-threshold dissociation; microwave dissociation; high harmonics generation; time-dependent quantum mechanics; Morse oscillator.

\section{Introduction}

It is well-known that a high-intensity, ultrafast infrared laser of wavelength, e.g., 1064 or $800 \mathrm{~nm}$, will ionize an atom or a molecule, due to multiphoton processes, by lifting the system from the ground state to the electronic continuum. ${ }^{1-10}$ Analogously, using a highintensity microwave laser, it should be possible to dissociate a diatomic molecule by lifting it from its ground vibrational state to the vibrational continuum. However, to the best of our knowledge, this has not been accomplished so far, either experimentally or theoretically. Earlier, the dissociation of diatomic molecules under intense laser fields, including Coulomb explosion, was achieved mainly through electronic motions involving electron excitation and ionization ${ }^{4,11-13}$ rather than exclusively through vibrational motions or excitations. On the other hand, microwave lasers had been employed to study the photoionization of Rydberg atoms as well as to study highly excited states of the hydrogen atom, ${ }^{14-19}$ i.e., by already taking the atom concerned close to its electronic continuum. An earlier study ${ }^{20,21}$ on the far-infrared dissociation of $I_{2}^{+}$had taken the molecular ion to a highly excited vibrational state near the vibrational continuum such that by using a microwave laser of energy $60 \mathrm{~cm}^{-1}(0.000273$ a.u.) only

\footnotetext{
\#Dedicated to Prof. N Sathyamurthy on his 60th birthday

*For correspondence
}

100-200 photons were needed to reach the vibrational continuum. However, in spite of the previous works, it is worthwhile to note that multiphoton interaction between vibrational motions and intense laser fields has still not received the attention it deserves.

In this work, we examine the possibility of microwave dissociation of a diatomic molecule starting from its ground state both theoretically and computationally. We also study other accompanying multiphoton processes such as high-harmonics generation (HHG), and above-threshold dissociation (ATD) which arise due to the intense microwave laser-molecule interaction. We study the dynamics of such interactions by explicitly considering only vibrational motions and not electronic or rotational motions in the $\mathrm{CO}$ molecule which was chosen because of its unusually high dissociation energy of $11.09 \mathrm{eV},{ }^{22}$ i.e., 0.407556 a.u. (see section 3.3 for the justification of neglecting rotational motions). Earlier, both field ionization and Coulomb explosion under intense femtosecond laser fields had been reported on this molecule, ${ }^{12,13}$ by considering electronic motions. Therefore, the question of ionization by microwave discharge in gases is also not considered in this paper.

The most appropriate, complete and accurate method for studying the nonrelativistic dynamical evolution of quantum systems under strong driving forces is the numerical solution of the corresponding timedependent Schrödinger equation (TDSE). Although this 
approach is computationally intensive, it has the advantage of not requiring any restriction on the nature of basis sets or the type of laser pulse used while the solutions can be obtained for varying intensities and frequencies. The computational labour can be significantly reduced by employing reliable and realistic one-dimensional models which are not only relatively easier to solve numerically but also permit one to conduct extensive numerical experiments for studying the dependence of various nonlinear multiphoton phenomena on different parameters. It has been established earlier $^{23,24}$ that one-dimensional nonlinear oscillators can reproduce the important characteristic features of atoms and molecules under intense laser fields. In the present work, the $\mathrm{CO}$ molecule was modelled as a Morse oscillator which is the most commonly used onedimensional model for vibrating diatomic molecules.

Section 2 of this paper describes the method of calculation. Section 3 discusses the results and conclusions are given in section 4 .

\section{Method of calculation}

The one-dimensional Morse oscillator potential is given by

$$
V(x)=D_{e}\left[1-\exp \left(-\alpha\left(x-x_{e q}\right)\right)\right]^{2},
$$

where $D_{e}$ is the well depth, $x$ is the internuclear distance, $x_{e q}$ is the bond length and $\alpha$ is a positive constant. Under the Born-Oppenheimer approximation, the vibrational levels of this system can be calculated by solving the nuclear Schrödinger equation involving the unperturbed Hamiltonian (atomic units)

$$
H_{0}=-(1 / 2 \mu)\left(d^{2} / d x^{2}\right)+V(x),
$$

where $\mu$ is the reduced mass of the molecule. For the CO molecule, we employ the following parameters: ${ }^{22}$

Morse frequency, $\omega_{\mathrm{e}}=2169.82 \mathrm{~cm}^{-1}=$ 0.00988643 a.u. Anharmonicity constant, $\chi_{\mathrm{e}}=$ 0.006126776. Equilibrium internuclear distance, $x_{e q}=2.1322$ a.u. Dissociation energy, $\mathrm{D}_{0}=11.09 \mathrm{eV}=$ 0.407556 a.u. $\mu=12589.049$ a.u. $\alpha=1.2349$ a.u.

In Equation 2, we have omitted rotational motions. Later, we will argue that such omission is justified.

When the linearly polarized laser field is applied, the Hamiltonian of the system becomes

$$
H=H_{0}+d(x) f(t) E_{0} \sin \left(\omega_{L} t\right),
$$

where $d(x)$ is the dipole moment operator, $E_{0}$ is the maximum field strength $\left(E_{0}=(8 \pi I / \mathrm{c})^{1 / 2}, I\right.$ being the laser intensity and $c$ the speed of light), $\omega_{L}$ is the incident laser frequency and the linear ramp function $f(t)=t / t_{0}$ up to three optical cycles and unity thereafter $\left(t_{0}=48256.45056\right.$ a.u. $\left.=1.125 \mathrm{ps}\right)$.

Ting ${ }^{11,25}$ as well as Goggin and Milonni ${ }^{26}$ had defined $d(x)$ as a linear function of distance. This makes the dipole moment increase with $x$ whereas it should actually decrease. Therefore, we adopt Tanner and Maricq's ${ }^{20,21}$ prescription of

$$
d(x)=x \exp \left[-x /\left(a x_{e q}\right)\right],
$$

where ' $a$ ' is a positive constant. A similar form was employed by Heather and Metiu. ${ }^{27,28}$ For the CO molecule, we have adopted $a=0.25638$, so that the experimental dipole moment of 0.04328 a.u. is reproduced as the expectation value of $d(x)$ with our calculated ground state.

For the $\mathrm{CO}$ molecule, the vibrational ground-state wavefunction is obtained by solving the nuclear TDSE in imaginary time by first transforming the TDSE into a diffusion quantum Monte Carlo (DQMC)-type equation. ${ }^{29}$ The system is then evolved in imaginary time until a global minimum corresponding to the stationary ground state is reached (for real-time dynamics, the ground state corresponds to $t=0$ ). The same procedure is to be followed to obtain the energy eigenvalues for excited states, by first ensuring that each state is orthogonal to all lower states. The detailed numerical methodology was reported earlier. ${ }^{30}$ This DQMC approach had successfully been employed earlier to obtain the energies of atomic and molecular systems ${ }^{31,32}$ as well as those of double-well, ${ }^{30}$ multiple-well ${ }^{33}$ and self-interacting ${ }^{34}$ nonlinear oscillators. Our calculated vibrational ground-state energy for the $\mathrm{CO}$ molecule, neglecting rotational contributions and using $\Delta x=0.01$ (the same spatial mesh would be employed for real-time dynamics as given below), is 0.00497 a.u. compared to the analytical Morse eigenvalue of 0.00493 a.u. However, note that using very fine spatial and temporal mesh as well as very low tolerance for convergence makes the numerical method exact in principle; ${ }^{30}$ but this demands much larger computer time.

The quantum dynamics of the vibrating $\mathrm{CO}$ molecule were then studied by using the same algorithm in real time, i.e., by numerically solving the TDSE in real time instead of solving a diffusion equation. The system is allowed to evolve for 3 ps over 8 optical cycles under an intense microwave laser with wavelength $\lambda_{L}=$ $116.65 \mu \mathrm{m}$, i.e., frequency $\omega_{L}=0.0003906$ a.u., with a time-step $(\Delta t)$ of 0.49089 a.u. so that one optical cycle is completed in 32768 time-steps. With the above laser frequency, 25 photons are required to excite the molecule (unperturbed Morse oscillator) from its ground vibrational to the first excited vibrational state. 
This numerical solution of the TDSE employed a large computation grid, $0 \leq x \leq 980$ a.u., with 98000 spacegrid points while for each laser intensity the total number of time-steps was 2,62,144. The justification for taking such a large space grid is that, if molecular dissociation is to be achieved, the probability density for the system under the influence of the laser field would have to spread fairly rapidly over a large distance and thus encroach into the vibrational continuum. Furthermore, the use of a large computation grid eliminates the necessity for using either an absorbing potential or a mask function to take care of reflections from the grid boundaries. The laser intensities employed were $1 \times 10^{13}$ and $5 \times 10^{18} \mathrm{~W} \mathrm{~cm}^{-2}$.

\section{Results and discussion}

\subsection{Time-dependent probability density and dissociation probability}

The normalized ground-state wavefunction is the initial state of the system at $t=0$. The corresponding probability density is nearly a Gaussian centred at $x_{e q}$ (figure 1a). With the onset of the laser field $(I=1 \times$ $10^{13} \mathrm{~W} \mathrm{~cm}^{-2}$ ) and subsequent progress of time, the probability density does not remain like a simple Gaussian and begins to spread spatially as seen in a wave packet. At $t=4.4182$ a.u., this spread is less and several new peaks, corresponding to contributions from excited states, appear in the probability density (figure $1 \mathrm{~b}$ ). The appearance of nodes and minima signify the presence of pure excited states as well as superposition of states. At $t=48.5981$ a.u., i.e., after only 99 time-steps, the probability density develops an accordion-like ${ }^{15}$ pattern of maxima, each maximum enveloping several peaks, the spatial extension of the wave packet being nearly 500 times that in the ground state (figure 1c). As higher and higher excited states, Stark-shifted states, dressed states and continuum states mix into the time-evolved wavefunction, the probability density becomes very complicated (figure 1d,e,f). Such a behaviour is expected from a system driven by strong external fields. As shown in figure $1 \mathrm{~d}$,e and $\mathrm{f}$, the probability density has wandered into the vibrational continuum, creating a final state (mixture of numerous states) which is dispersed over a large spatial region and has a reduced norm $N(t)=\langle\psi(x, t)| \psi(x, t)>$, compared to the initial $(t=0)$ norm of unity. Furthermore, as time progresses, earlier larger peaks (figure 1) subside while the peaks near the grid-end increase in height, indicating smaller proportions of the ground and lower excited states compared to the higher states in the time-evolved
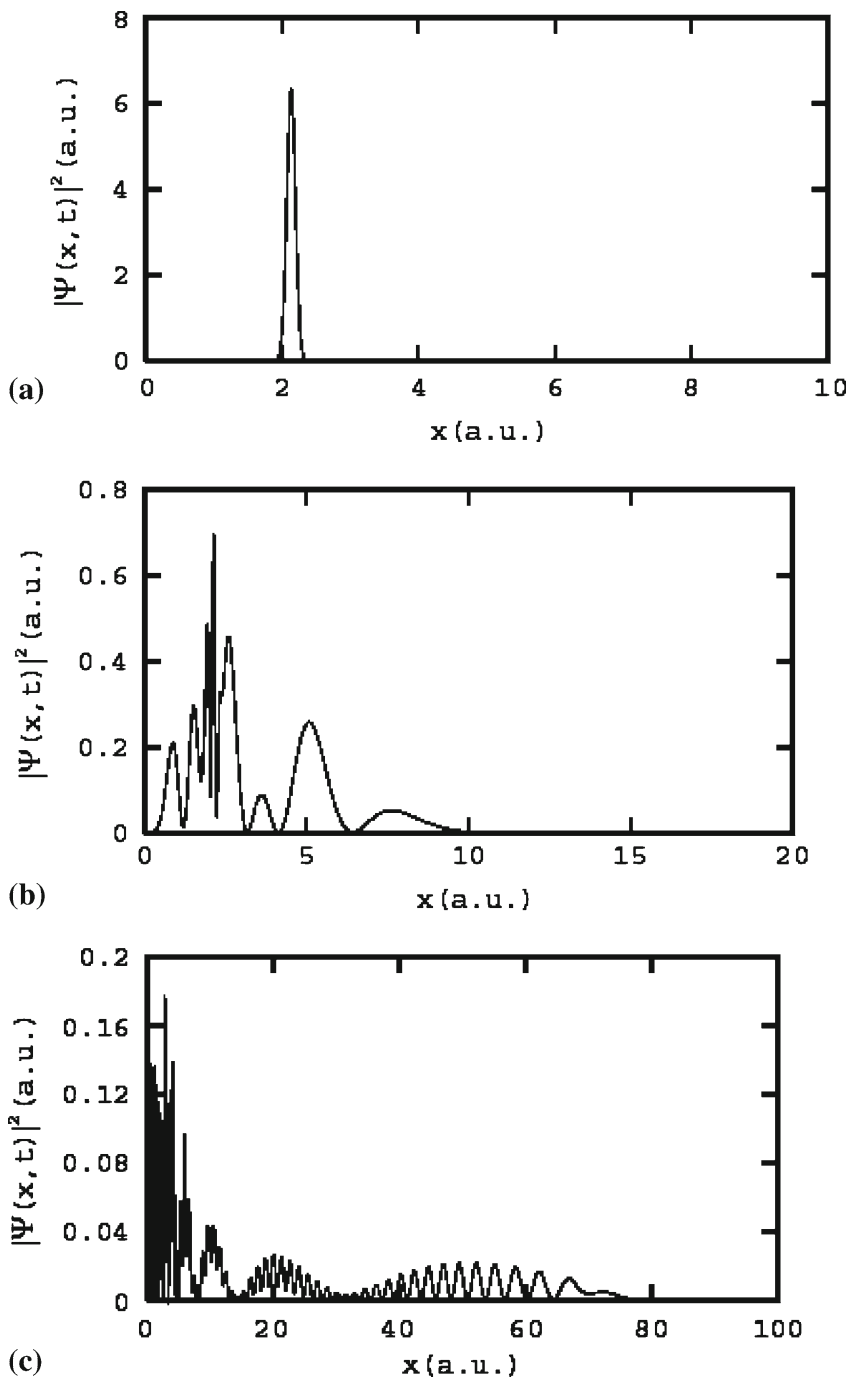

Figure 1. Time-dependent probability density $\left(|\psi(x, t)|^{2}\right)$ in a.u. for (a) $t=0$, (b) $\mathrm{t}=4.4182$, (c) $t=48.5982$ a.u., respectively. (d), (e) and (f) refer to the crest $\left(E=E_{0}, t=\right.$ 116620.099 a.u.), center ( $E=0, t=120641.482$ a.u.) and trough ( $E=-E_{0}, t=124662.864$ a.u.) respectively of the 8th optical cycle. $I=5 \times 10^{18} \mathrm{~W} \mathrm{~cm}^{-2}$.

wavefunction (all the peaks fluctuate with time). Separately and together, these features indicate the onset of dissociation.

The dynamics of dissociation can be explicitly studied through the time-dependent dissociation probability, $P_{\text {diss }}(t)$. Although there have been several definitions of $P_{\text {diss }}(t)$ in the literature, ${ }^{11,20,21,27,28}$ we adopt the definition ${ }^{25}$

$$
P_{\text {diss }}(t)=1-N(t)
$$

in view of our deploying a large spatial grid. This definition is appropriate since, with the probability density leaking into the continuum, the norm $N(t)$ decreases in time. This can also be regarded as the onset of dissociation because unless the density wanders into the continuum, the norm should remain unity. Furthermore, the 

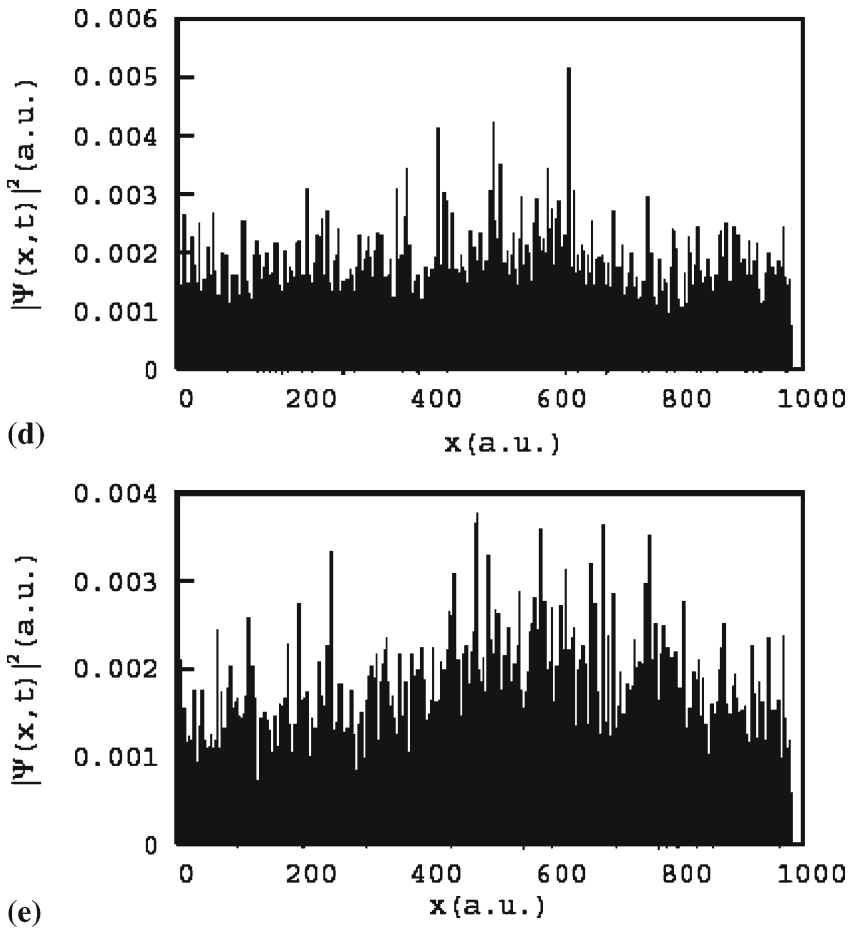

(e)

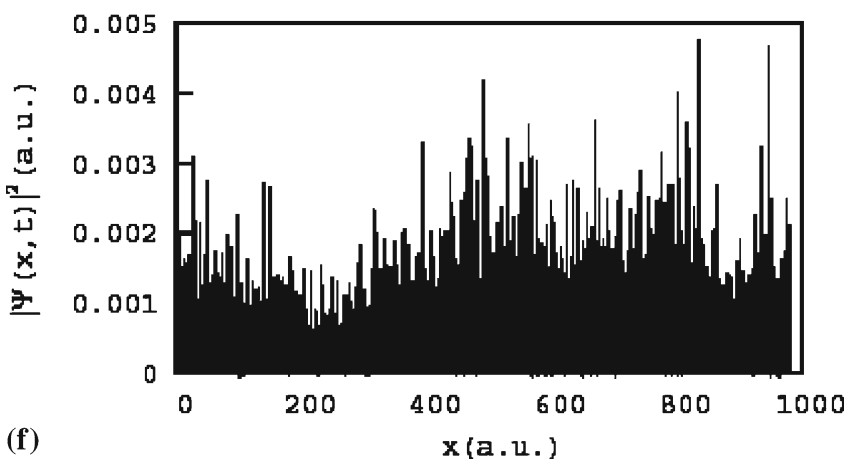

Figure 1. (continued)

TD potential (see below) $U(x, t)$ attains a constant value $D_{e}$ at $x=18.2$ a.u. for both the laser intensities. Obviously, one must interpret the probability density spreading beyond $x=18.2$ a.u. as its gradual leaking into the continuum.

Figure 2 shows the plot of $P_{\text {diss }}(t)$ as a function of time-steps, for $I=1 \times 10^{13} \mathrm{~W} \mathrm{~cm}^{-2}$ (a similar plot is obtained for $I=5 \times 10^{18} \mathrm{~W} \mathrm{~cm}^{-2}$ ). After an initial induction period, $P_{\text {diss }}(t)$ increases and proceeds in steps. For a single molecule, such a step-wise dissociation phenomenon does not appear to have been encountered before. After 8 optical cycles, $\sim 60 \%$ dissociation was achieved.

It is well-known that a system, under interaction with an intense laser of given wavelength and intensity, is surrounded by billions of photons (e.g., a 1064 or $800 \mathrm{~nm}$ laser of intensity $10^{14} \mathrm{~W} \mathrm{~cm}^{-2}$ has a photon density of the order of $10^{10}$ photons per cubic wavelength ${ }^{9}$ ),

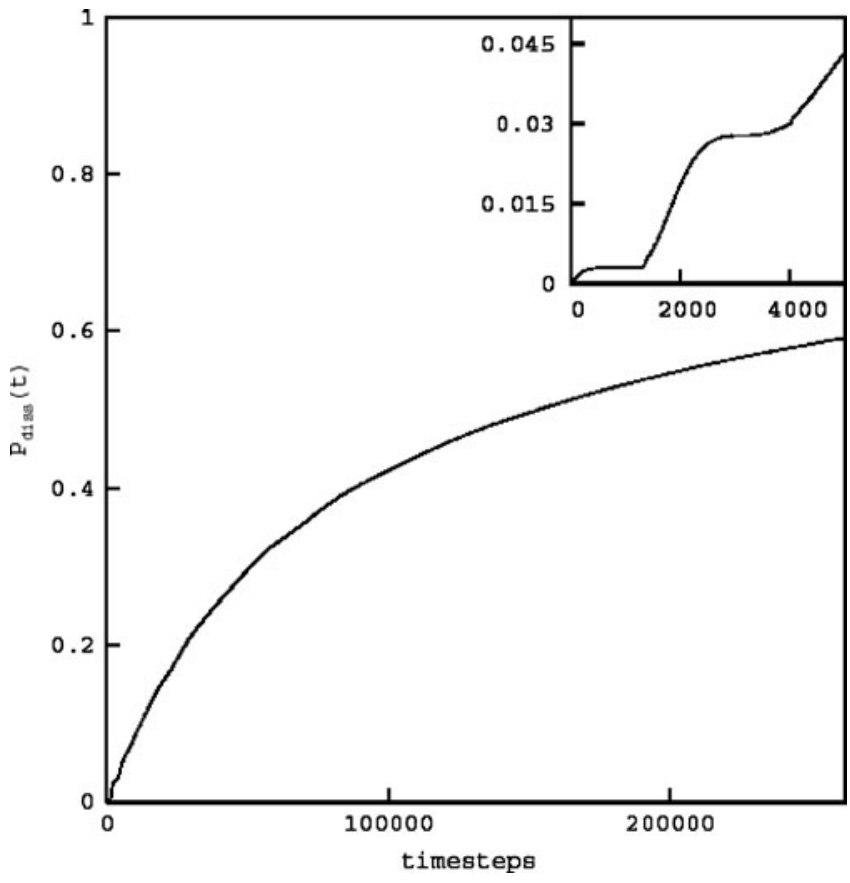

Figure 2. The dissociation probability, $P_{\text {diss }}(t)$ as a function of time-steps, in a.u., for $I=1 \times 10^{13} \mathrm{~W} \mathrm{~cm}^{-2}$. The inset shows the plot for the first 5000 time-steps.

The minimum number, $n$, of photons necessary to just lift the molecule to the lowest edge of the vibrational continuum is given by

$$
n=D_{0} / \omega_{L}=1044 .
$$

Figure 2 shows that the significant dissociation begins after a preliminary 'induction period' of $t \approx 640$ a.u. or $14.9 \mathrm{fs}$. This indicates that the molecule has been lifted into the continuum (ATD).

\subsection{The time-dependent potential}

For the present problem, the TD potential is given by

$$
U(x, t)=V(x)+d(x) f(t) E_{0} \sin \left(\omega_{L} t\right),
$$

which reduces to the unperturbed Morse potential $V(x)$ when $t=n \pi / \omega_{L}, n=0,1,2, \ldots$ etc. At all other times, $U(x, t)$ is drastically modified by the intense laser field. Earlier, we had argued ${ }^{23}$ that selection rules for spectral transitions, derived from static considerations, are unlikely to be valid in such situations. Figure $3 \mathrm{a}$ and $\mathrm{b}$ depict $U(x, t)$ as a function of $x$ at $t=0$ as well as at the crest and trough of the 8th optical cycle for $I=5 \times 10^{18} \mathrm{~W} \mathrm{~cm}^{-2}$ respectively. Note that for $I=1 \times 10^{13}$ and $5 \times 10^{18} \mathrm{~W} \mathrm{~cm}^{-2}$, the $E_{0}$ values are 0.01688 and 11.936 a.u., respectively. A plot of $U(x, t)$ when $\sin \left(\omega_{L} t\right)$ is unity has the same appearance as the unperturbed Morse potential although the 
well-depth is affected by the magnitude of $E_{0}$ with the well shifted to the left or right for the trough or crest, respectively. Figure $3 \mathrm{~b}$ indicates a rather shallow minimum for $U(x, t)$ when $E=E_{0}$ and $f(t)=1$, indicating that at a higher laser intensity this curve would be repulsive while the potential minimum would become deeper, shifting further to the left when $E=-E_{0}$ and $f(t)=1$.

\subsection{The HHG and ATD spectra}

The TD dipole moment is given by

$$
d(t)=<\Psi(x, t)|d(x)| \Psi(x, t)>,
$$

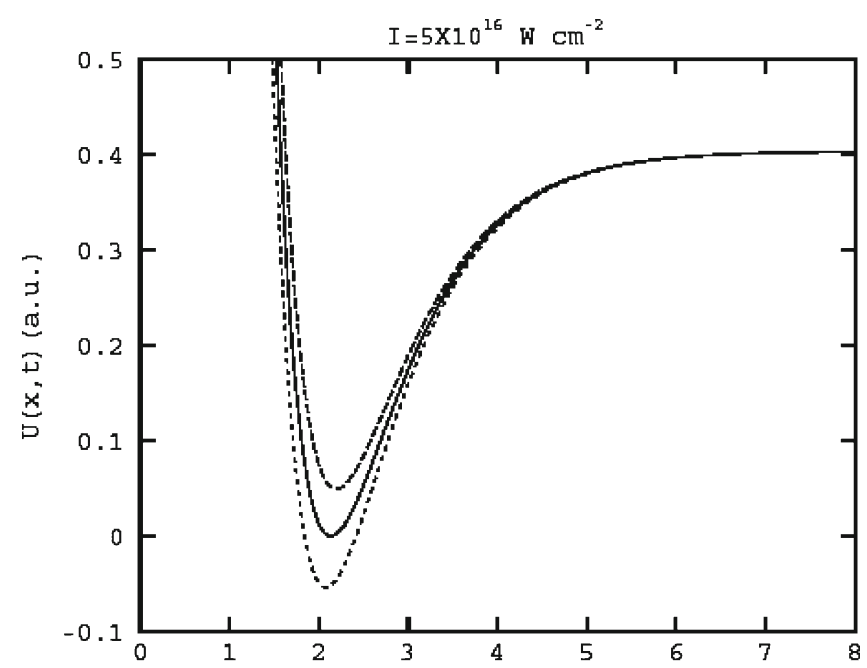

(a)

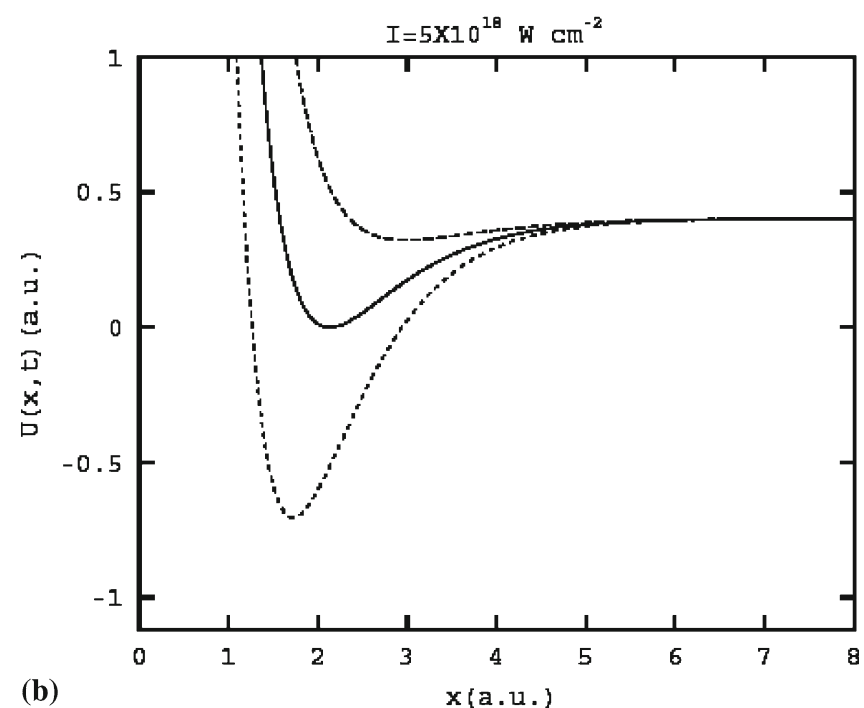

Figure 3. The time-dependent potential $U(x, t)$ as a function of $x$, in a.u., for $I=5 \times 10^{18} \mathrm{~W} \mathrm{~cm}^{-2}$. The solid line refers to the unperturbed Morse potential, the dashed line above the Morse potential refers to the crest $\left(E=E_{0}\right)$ while the dotted line below the Morse potential refers to the trough ( $\left.E=-E_{0}\right)$ respectively of the 8th optical cycle. where $d(x)$ is given by Equation 4. As a result of interaction with the laser field, the initial dipole moment changes into a complicated pattern whose periodicity is twice that of the laser field (figure 4). Towards the latter part of the interaction, $d(t)$ is quite small, indicating dissociation. Figure 5 depicts the HHG spectra obtained by taking a fast Fourier transform (FFT) $D(\omega)$ of $d(t)$ over the last four optical cycles. As expected, the spectra have the characteristic pattern of the atomic and molecular HHG spectra, viz., a rapid decay of peak intensities for the first few harmonics, followed by a plateau of harmonics with comparable intensities and then a sharp fall in harmonic intensities (figure 5). Note that here we are not considering the electronic dipole moment which is more effective than $d(t)$ above in generating high-order harmonics. Therefore, in figure 5, the HHG spectra are relatively feeble in intensity even though the spectrum is quite complex with distinct plateaus. The first plateau is associated with the vibrational ground state whereas the appearance of lower plateaus indicates dissociation. The inset of figure 5a, with $I=1 \times 10^{13} \mathrm{~W} \mathrm{~cm}^{-2}$, shows odd, even and shifted harmonics while the overall spectrum is quite dense with such harmonics. Such a phenomenon has
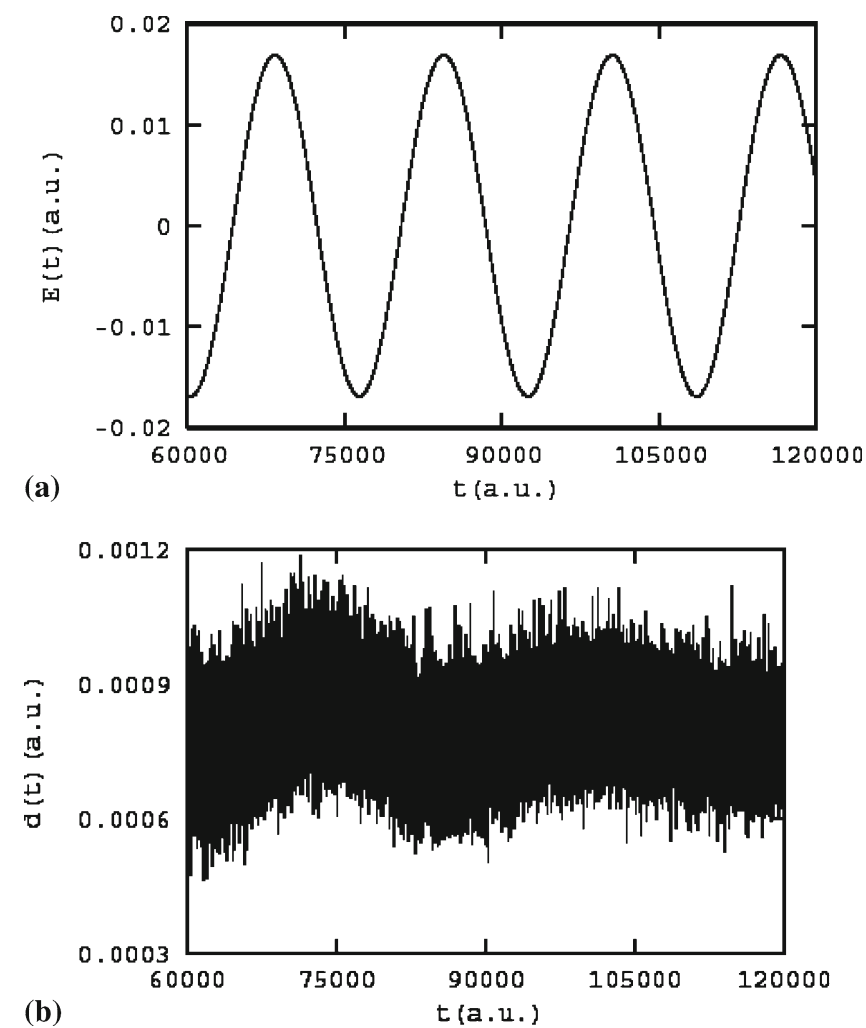

Figure 4. Time-dependent laser electric field $E(t)$ and dipole moment $d(t)$, as functions of time, in a.u. (a) and (b) correspond to $I=1 \times 10^{13} \mathrm{~W} \mathrm{~cm}^{-2}$ while $(\mathbf{c})$ and (d) correspond to $I=5 \times 10^{18} \mathrm{~W} \mathrm{~cm}^{-2}$, respectively. 


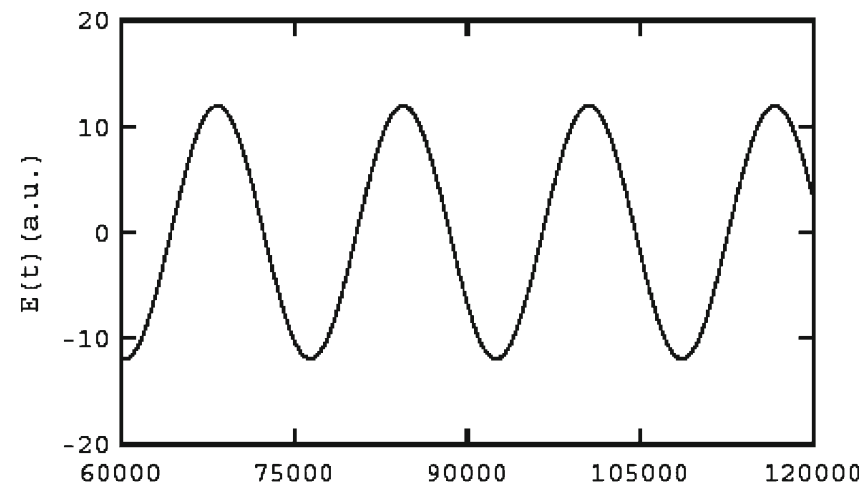

(c)

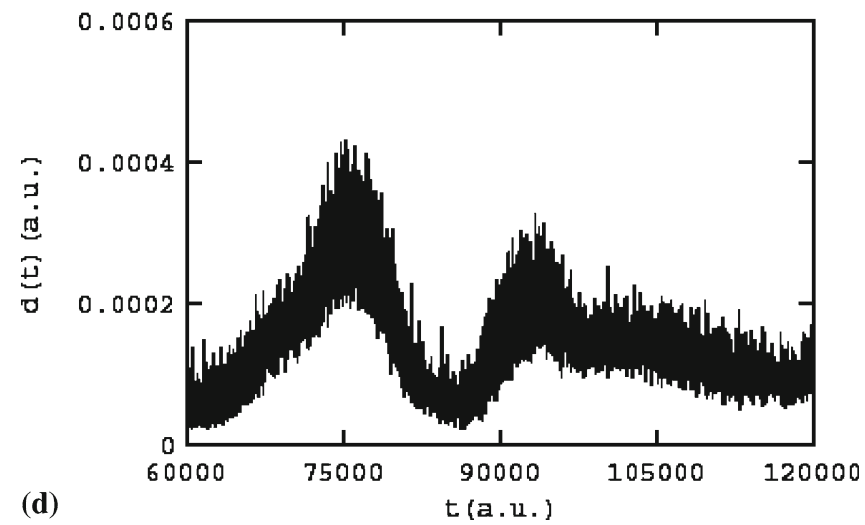

Figure 4. (continued)

been noted earlier ${ }^{23,24}$ with HHG spectra generated by nonlinear oscillators. The appearance of both odd and even harmonics is due to the fact that the Morse potential is neither symmetric nor antisymmetric in $x$. Here, the main reasons for the shifted harmonics are likely to arise from the Stark effect rather than parity violation. ${ }^{23}$

It is worthwhile to speculate upon the possible effects of molecular rotation on the HHG spectra. At first sight, it might appear that due to rotations of the molecular dipole its interactions with the laser electric field might be hindered, thereby delaying the transition of the molecule to the vibrational continuum and consequently affecting the HHG spectra. However, such a delay is quite unlikely to alter the characteristic pattern of the HHG spectra. Furthermore, since the transition from the ground vibrational state to the continuum takes place in femtoseconds, by the time the molecule completes one cycle of rotation, it would already be in the continuum. In other words, the much slower rotational motion is unlikely to affect the HHG spectra. Additionally, because of centrifugal distortion, rotation ought to facilitate molecular dissociation, thereby partly countering the orientation effects of the molecular dipole.

Figure 6 shows the vibrational ATD spectrum obtained by taking an FFT $A(\omega)$ of the autocorrelation function $<\psi(x, t) \mid \psi(x, t=0)>$ over the last
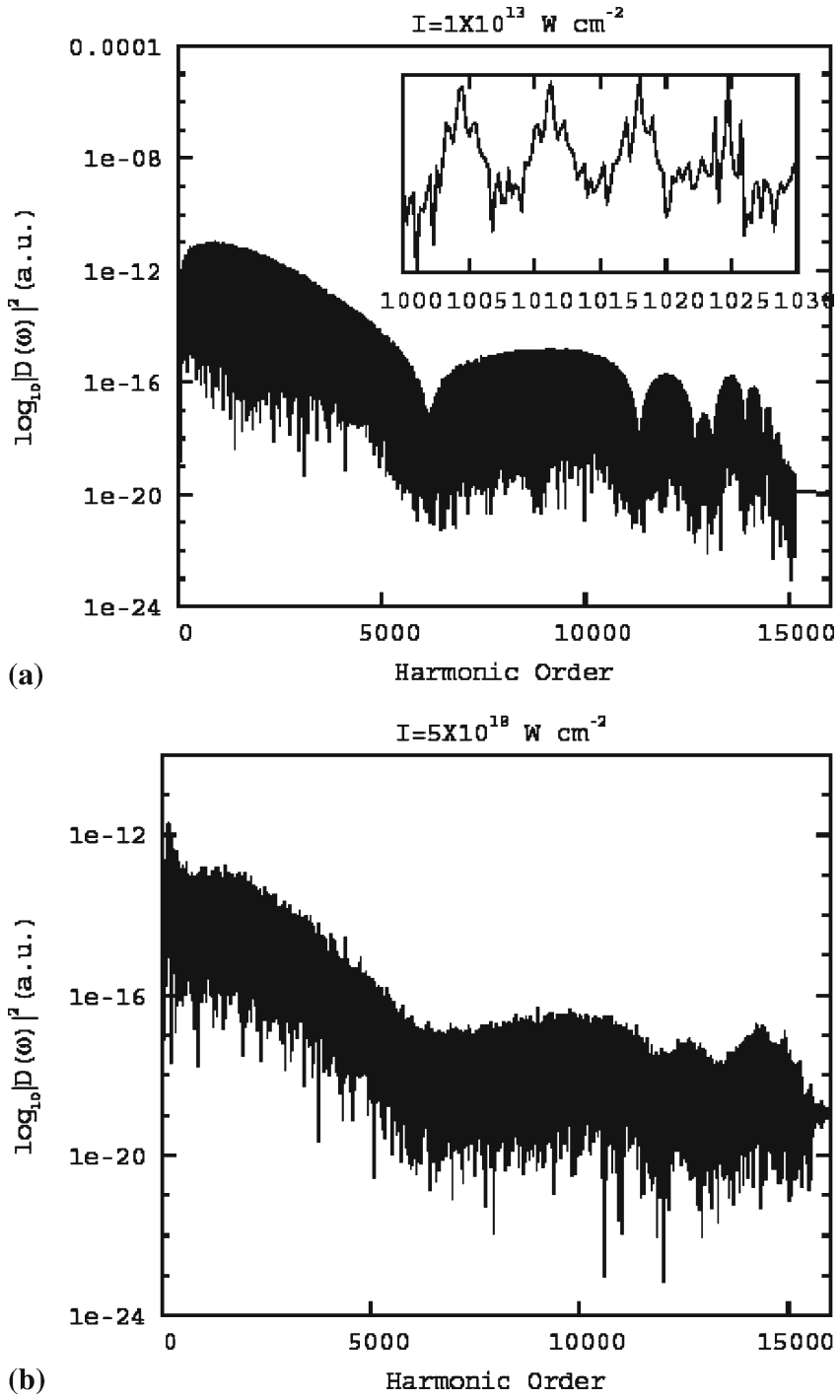

Figure 5. Vibrational HHG spectra (a.u.) at (a) $I=1 \times$ $10^{13} \mathrm{~W} \mathrm{~cm}^{-2}$ and (b) $I=5 \times 10^{18} \mathrm{~W} \mathrm{~cm}^{-2}$. Inset of (a) shows odd, even and shifted harmonics.

four optical cycles. In cases involving molecular electronic motions, such an FFT generates above-threshold ionization (ATI) which may then be followed by ATD. In contrast, the present FFT has no ATI component. At $I=1 \times 10^{13} \mathrm{~W} \mathrm{~cm}^{-2}$ (figure 6a), there is a single prominent peak of height $\sim 3.7 \times 10^{-4}$ near $\omega \sim 0.4$, i.e., near the dissociation limit, whereas at $I=5 \times 10^{18} \mathrm{~W} \mathrm{~cm}^{-2}$ one observes many peaks of relatively lower heights (figure 6b). Each of the humps in figure 6b comprises a series of peaks. The humps in the ATD spectrum clearly correspond to those present in the respective HHG spectrum (figure 5b) because both spectra originate from the continuum. Indeed, the smaller humps in the ATD spectrum are attributable to higher continuum states. Note that such 'pure' vibrational ATD spectra, without the corresponding ATI, do not appear to 

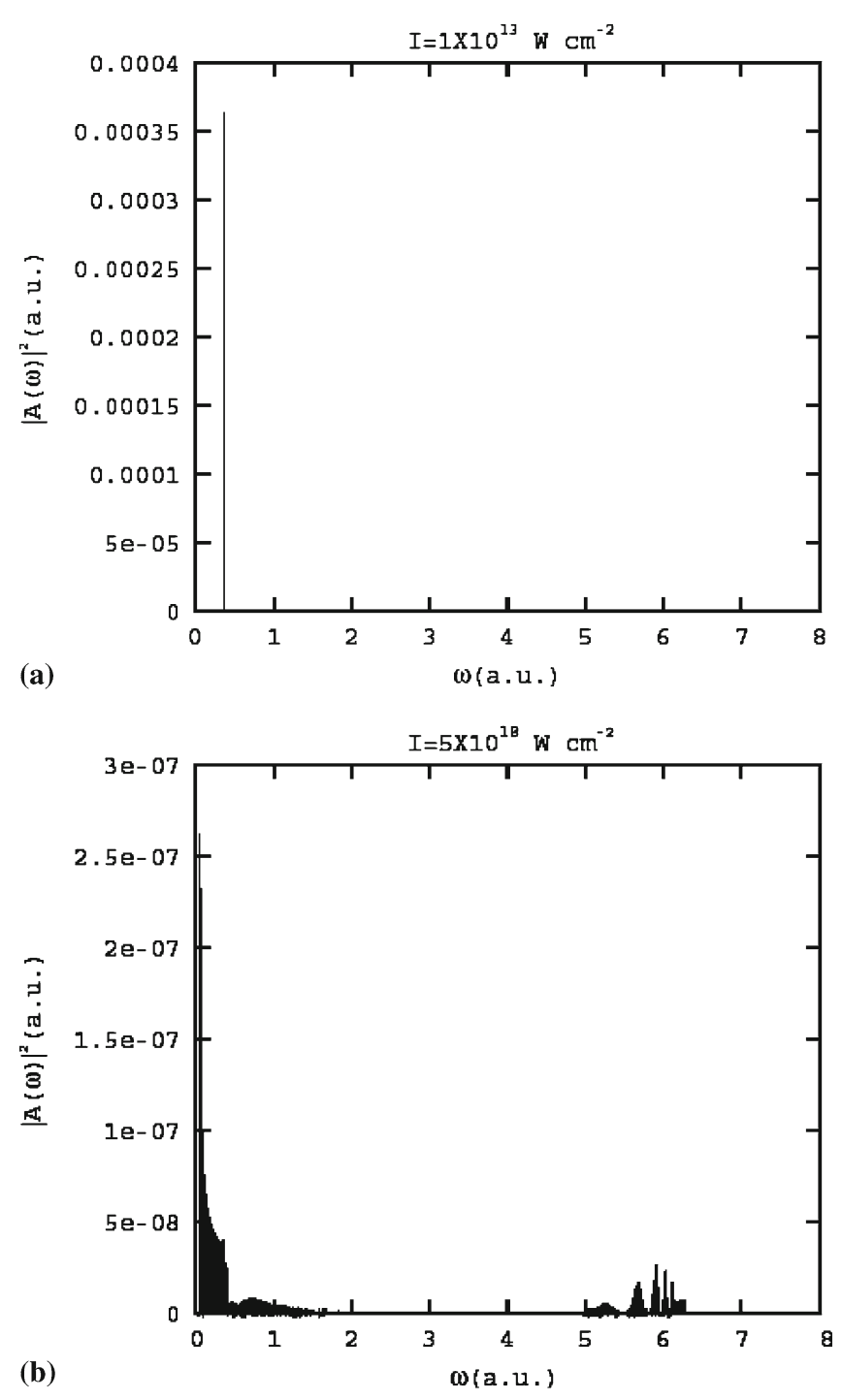

Figure 6. Vibrational ATD spectra, in a.u., at (a) $I=1 \times$ $10^{13} \mathrm{~W} \mathrm{~cm}^{-2}$, and (b) $I=5 \times 10^{18} \mathrm{~W} \mathrm{~cm}^{-2}$.

have been experimentally observed as yet, presumably because such intense microwave lasers are yet to be realized experimentally. ${ }^{35,36}$

\section{Conclusion}

The time-dependent quantum mechanical study reported in this paper leads to the prediction that the $\mathrm{CO}$ molecule can be dissociated by a microwave laser of the appropriate intensity (e.g., $I \geq 1 \times 10^{13} \mathrm{~W} \mathrm{~cm}^{-2}$ for $\lambda_{L}=116.65 \mu \mathrm{m}$ ). The absorption of 1044 photons would lift the molecule to the lower edge of the vibrational continuum from which it can undergo abovethreshold dissociation. All the quantities examined in this paper such as the time-dependent probability density, dissociation probability, norm, potential function, HHG and ATD spectra, etc. are consistent with respect to such dissociation. Therefore, provided such lasers are available and appropriate experimental conditions can be realized in the laboratory, microwave radiation is predicted to break chemical bonds, even selectively.

\section{Acknowledgements}

A major part of this work was carried out when AW was in Panjab University, Chandigarh, India. AW thanks Council of Scientific and Industrial Research (CSIR), New Delhi, for financial support during the period.

\section{References}

1. Gavrila M (Ed.) 1992 Atoms in intense laser fields (New York: Academic Press)

2. Mittleman M H 1993 Introduction to the theory of laseratom interactions (New York: Plenum Press)

3. Faisal F H M 1987 Theory of multiphoton processes (New York: Plenum Press)

4. Bandrauk A D (Ed.) 1994 Molecules in laser fields (New York: Marcel Dekker)

5. Burnett K, Reed V C and Knight P L 1993 J. Phys. B 26 561

6. Protapapas M, Keitel C H and Knight P L 1997 Rep. Prog. Phys. 60389

7. Joachain C J, Dorr M and Klystra N J 2000 Adv. At. Mol. Opt. Phys. $\mathbf{4 2} 225$

8. Eberly J H, Grobe R, Law C K and Su Q $1992 A d v$. At. Mol. Opt. Phys. Suppl. 1301

9. Eberly J H and Kulander K C 1993 Science 262129

10. Yamanouchi K, Chin S L, Agostini P and Ferrante G (eds) 2007 - 2010 Progress in ultrafast intense laser science Vols. 1-4 (New York: Springer)

11. Ting J J-L 1994 J. Phys. B 271249

12. Ren H, Mia R, Chen J, Li X, Yang H and Gong Q 2003 J. Phys. B 362179

13. Hatten D L, Zhu J, Goldbar J and Hill III W T 1997 Laser Phys. 71

14. Van Leeuwen K A H, Van Oppen G, Renwick S, Bowlin J B, Koch P W, Jensen R V, Rath O, Richards D and Leopold J G 1985 Phys. Rev. Lett. 552231

15. Leopold J G and Richards D 1985 J. Phys. B 183369

16. Jensen R V 1984 Phys. Rev. A 30386

17. Blumel R and Smilansky P 1987 Phys. Rev. Lett. 582531

18. Casati G, Chirikov B V and Shepelyansky D L 1984 Phys. Rev. Lett. 532525

19. Casati G, Chirikov B V, Guarneri I and Shepelyansky D L 1989 Phys. Rev. Lett. 592927

20. Tanner J J and Maricq M M 1988 Chem. Phys. Lett. 149 503

21. Tanner J J and Maricq M M 1989 Phys. Rev. A 404054

22. Berry R S, Rice S A and Ross J 2000 Physical chemistry (Oxford: Oxford University Press) p. 189

23. Wadehra A, Vikas and Deb B M 2003 J. Chem. Phys. 1196620

24. Wadehra A and Deb B M 2003 Proc. Indian Acad. Sci. (Chem. Sc., C.N.R. Rao Festschrift) 115 349; erratum 2004 J. Chem. Sci. 116129

25. Ting J J-L 1995 Phys. Rev. A 512641 
26. Goggin M E and Milonni P W 1988 Phys. Rev. A 37796

27. Heather R and Metiu H 1987 J. Chem. Phys. 865009

28. Heather R and Metiu H 1988 J. Chem. Phys. 885496

29. Hammond B L, Lester Jr. W A and Reynolds P J 1994 Monte Carlo methods in ab initio quantum chemistry (Singapore: World Scientific Press)

30. (a) Roy A K, Gupta N and Deb B M 2002 Phys. Rev. A 65 012109; (b) Gupta N, Wadehra A, Roy A K and Deb B M 2001 In recent advances in atomic and molecular physics (ed.) R Srivastava (New Delhi: Phoenix Press)

31. Dey B K and Deb B M 1999 J. Chem. Phys. 1106229
32. Roy A K, Dey B K and Deb B M 1999 Chem. Phys. Lett. 308523

33. Gupta N, Roy A K and Deb B M 2002 Pramana-J. Phys. 59575

34. Wadehra A, Roy A K and Deb B M 2003 Int. J. Quantum Chem. 91597

35. Orzechowski T J 2005 www.osti.gov/bridge/servlets/ purl/6214813

36. Pinhasi Y, Lurie Yu and Yahalom A 2005 In Quasioptical control of intense microwave transmission (eds) J L Hirschfield and M I Petelin (Berlin: Springer) p. 253 